Rev. salud pública. 14 (2): 282-295, 2012

\title{
Estimación de la fuerza de infección de Hepatitis A en Colombia, aplicando modelos catalíticos
}

\section{Assessing the force of hepatitis A virus infection in Colombia by applying catalytic models}

Carlos J. Rincón ${ }^{1}$, Nelcy Rodríguez-Malagón ${ }^{2}$, Cristina Mariño ${ }^{3}$, Jose A. Mojica ${ }^{4}$ y Fernando de la Hoz-Restrepo ${ }^{5}$

1 Instituto de investigaciones Clínicas, Facultad de Medicina. Universidad Nacional de Colombia. Universidad de La Sabana-Área de Investigación. Bogotá, Colombia. cjrinconr@bt.unal.edu.co

2 Departamento de Estadística, Universidad Nacional de Colombia. Departamento de Epidemiología Clínica y bioestadística, Pontificia Universidad Javeriana. Bogotá, Colombia. nrodriguezm@, unal.edu.co

3 Hospital Militar Central, Área de Pediatría. Bogotá, Colombia. cristina.marino@gmail.com

4 Sanofi Pasteur-Vacunas. Bogotá, Colombia. josealejandro.mojica@sanofi.com

5 Departamento de Salud Pública, Facultad de Medicina. Universidad Nacional de Colombia. Bogotá, Colombia.fpdelahoz@yahoo.com.ar

Recibido 25 Mayo 2011/Enviado para Modificación 8 Marzo 2012/Aceptado 9 Abril 2012

\section{RESUMEN}

Objetivo Estimar la fuerza de infección de hepatitis $A$ en la población de niños entre 1 a 15 años de edad que asistieron a seis centros de salud en Colombia entre el año 2007 y 2008, aplicando modelos catalíticos.

Métodos En 2152 pacientes de seis centros de salud en cinco ciudades de Colombia se estimó la seroprevalencia de anticuerpos contra el virus de la Hepatitis A (anti-VHA) por grupos de edad. Con base en esta estimación, se obtuvo la fuerza de infección y la edad promedio de infección para cada región ajustando modelos catalíticos.

Resultados La fuerza de infección fue de 0,15 en el grupo de 1 a 4 años en Barranquilla. En el resto de ciudades la fuerza de infección tomó valores entre 0,02 para Bogotá en el grupo de 5 a 15 años, y 0,06 para Medellín en el grupo de 1 a 9 años. La edad promedio de infección en Bogotá, Bucaramanga, Cali y Medellín estuvo entre 10,7 y 12,0 años.

Conclusión En Bogotá, Bucaramanga, Cali y Medellín se estimaron seroprevalencia de anti-VHA entre $26,3 \%$ y $40,4 \%$ en el grupo de 10 a 15 años, con una edad promedio de infección entre 10,7 y 12,0 años; comportamiento semejante a la de regiones en desarrollo con endemicidad intermedia.

Palabras Clave: Hepatitis A, prevalencia, modelos estadísticos (fuente: DeCS, BIREME). 


\section{ABSTRACT}

Aims Estimating the force of hepatitis A virus (HAV) infection concerning a 1- to 15 -year-old child population being attended at six healthcare centres in Colombia by applying catalytic models.

Methods Anti-HAV seroprevalence was estimated in 2,152 patients attending six health centres in 5 Colombian cities; based on such estimation, the force of infection and average age of infection were obtained for each region.

Results The 1- to 4-year-old age group's force of infection was 0.15 in Barranquilla; for the other cities the force of infection was 0.02 in Bogotá for the 5- to 15-year-old age group and 0.06 in Medellin for the 1- to 9-year-old age group. Average infection age in Bogotá, Bucaramanga, Cali and Medellin was 10.68 to 11.97 years-old.

Conclusion There was high anti-HAV prevalence in the young-adult population, average infection age being 10.69 to 11.97 years-old, thereby presenting a similar pattern to that of developing regions having intermediate level of endemicity.

Key Words: Hepatitis A, prevalence, models, statistical statistical model (source: $\mathrm{MeSH}, \mathrm{NLM})$..

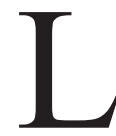

a hepatitis A es una enfermedad que ha sido documentada desde el siglo XVII, pero su etiología viral sólo fue postulada y posteriormente confirmada en 1940 y 1944 respectivamente (1). Es una enfermedad infectocontagiosa, asociada con malas condiciones sanitarias y de pobreza $y$ se transmite al contacto con las heces de otra persona que tenga el virus (vía fecal-oral), al consumir líquidos o alimentos contaminados o a través de vectores como moscas o cucarachas. La hepatitis A es una enfermedad de curso autolimitado, cuyo reservorio es el ser humano y su sitio de replicación es el hígado. La enfermedad clínica suele ser leve o asintomática en grupos de menor edad y no produce hepatitis crónica; en raras ocasiones causa una hepatitis fulminante lo que se refleja en una baja tasa de mortalidad del 0,1\% (1). Al inicio de la infección, aparecen anticuerpos específicos de tipo $\operatorname{IgM}$, presentándose generalmente valores elevados entre la primera y la sexta semana encontrándose casos (13\%) de hasta seis meses con este anticuerpo (2). La presencia de anticuerpos de tipo IgG indica que ya se ha presentado la infección por Hepatitis A y la persona tienen inmunidad de por vida $(3,4)$.

Con base en los resultados de seroprevalencia contra el virus de la hepatitis A por edad de la población, la Organización Mundial de la Salud (OMS) ha definido tres patrones de comportamiento de la infección, que se encuentran asociados con el nivel de desarrollo del país o región bajo estudio (5). Se considera una región de alta endemia cuando se 
observan seroprevalencia cercanas al $90 \%$ en la población de niños menores a los 10 años, donde se espera que en adultos la totalidad de la población sea inmune frente a la enfermedad. En estas regiones la OMS no recomienda realizar programas de vacunación. Endemia intermedia, cuando se observan seroprevalencia cercanas al $80 \%$ en la población de jóvenes adultos al cumplir 25 años de edad, indicando una mayor edad promedio de infección y donde la mayoría de adultos son inmunes frente a la enfermedad; este comportamiento se presenta en países en desarrollo. La OMS recomienda para estas regiones realiza vacunación en toda la población infantil junto con política de saneamiento ambiental. Por último se encuentran los países desarrollados donde se consideran de endemia baja cuando presentan bajos porcentaje de infección en los grupos de menor edad y un alto porcentaje de población adulta susceptible de contraer el virus. En estos casos, la OMS recomienda programas de vacunación en poblaciones con mayor riesgo de contagio.

La OMS estima un total anual de 1.4 millones de casos clínicos de hepatitis A en el mundo. En los Estados Unidos en las décadas de los 80 y 90 , se reportaron en promedio 26000 casos de hepatitis A al año, que representan una estimación de 270000 infecciones al incluir casos no reportados e infecciones asintomáticas (6). En Latinoamérica se estimó la seroprevalencia de anticuerpos contra el virus de la hepatitis A (anti-VHA) por grupos de edad (5) en seis países, encontrando que con excepción de algunos grupos de edad en Argentina y Venezuela, a mayor edad mayor seroprevalencia de anti-VHA. También se observa que a los 15 años, más del $50 \%$ de la población tenía anticuerpos presentes contra el virus de la hepatitis $\mathrm{A}$, siendo la prevalencia más alta en República Dominicana (90\%), seguida por México (88\%), Chile (70\%), Venezuela $(62 \%)$ y Brasil $(60 \%)$.

En la ciudad de Montevideo (Uruguay) (3) en una muestra de 896 niños que asistieron a cita de control o consulta médica a dos instituciones de salud (una privada y otra pública) se encontró una seroprevalencia global de infección del virus de hepatitis A del $26,7 \%$, observando que en los grupos de 2 a 6 años la seroprevalencia fue del $16,5 \%$, entre 7 y 11 años fue de 29,6 \% y de 12 a 14 años fue del 39,2 \%. En Lima (Perú) (7) de 859 personas que asistieron a dos instituciones de salud, se estimó una seroprevalencia global de anti-VHA de 65,2\%. 
Pese a que hay algunos estudios basados en población en Latinoamérica y que estos muestran que la endemicidad ha ido cambiando de alta a intermedia, aun no se utiliza la vacunación como una medida de prevención en la mayoría de los países. Esto se debe al desconocimiento parcial de la epidemiología del VHA, especialmente en factores como el número reproductivo básico, la edad promedio de infección y la proporción de población que debería vacunarse para disminuir los brotes. El presente estudio tuvo como objetivo estimar la fuerza de infección de hepatitis A en varias ciudades de Colombia aplicando modelos catalíticos utilizando una encuesta serológica realizada de 2007 a 2008 en 5 ciudades capitales del país.

\section{MATERIALES Y MÉTODOS}

Para el desarrollo de este trabajo se utilizó la base de datos recolectada en el proyecto: "Estudio piloto de seroprevalencia de Hepatitis A en niños y adolescentes (de 1-15 Años) en 5 ciudades colombianas", realizado por el Hospital Militar Central de Bogotá y el laboratorio Sanofi Pasteur, en el cual se capturó información de niños entre 1 y 15 años de edad que asistieron entre el año 2007 y 2008 a seis instituciones de salud ubicadas en cinco ciudades del país: Barranquilla $(n=380)$, Bogotá $(n=623)$, Bucaramanga $(n=409)$, Cali $(n=422)$ y Medellín $(n=422)$. En el anterior estudio, la selección de los seis centros de salud fue por conveniencia con los siguientes criterios de selección de la muestra:

Criterios de inclusión: Edad entre 12 meses a 15 años y consentimiento informado firmado por el padre, la madre o tutor legal.

Criterios de exclusión: Niños que hubieran presentado un cuadro de hepatitis, niño ya vacunado contra la Hepatitis A, niños con enfermedad evolutiva moderada o severa, esencialmente infecciones gastrointestinales o pacientes que consulten por síntomas como vómito y dolor abdominal o fiebre ( $\mathrm{T}^{\mathrm{o}}$ axilar $\geq 38^{\circ} \mathrm{C}$ ) sin foco al examen, coagulopatía no controlable, hepatoesplenomegalia el día de la consulta, historia clínica previa de ictericia (se excluye la ictericia neonatal $<10$ días), deficiencia inmunológica conocida (inclusive infección por HIV), historia previa de tratamiento con inmunosupresores, inclusive corticoterapia durante largos períodos $(>2$ semanas) durante el último mes y administración previa de inmunoglobulinas o productos derivados de la sangre durante los últimos 6 meses. 
Las variables observadas fueron: Presencia de anti-VHA (Positivo o negativo), edad, sexo, tipo de vivienda (casa, apartamento o inquilinato), número de adultos convivientes mayores de 15 años, número de niños convivientes menores de 15 años, zona de la vivienda (rural o urbana), estrato socioeconómico, número de dormitorios del lugar de residencia, si la vivienda cuenta con alcantarillado u otro tipo de disposición de excretas y si la vivienda cuenta con acueducto.

Para determinar la presencia de anti-VHA, el estudio piloto utilizó la pruebaAxSYM HAVAB ${ }^{\circledR} 2.0$ porAxSYM System de Abbott. Su preparación y procesamiento de las muestras, así como la interpretación de los resultados fue realizado teniendo en cuenta las instrucciones del fabricante.

Población y estimación de seroprevalencia de anti-VHA

Con base en los registros capturados en el estudio piloto, se realizó la descripción de cada una de las variables observadas y se estimó la seroprevalencia de anticuerpos contra el virus de la hepatitis A para cada ciudad por sexo, grupos de edad y estrato socioeconómico.

Fuerza de infección y modelos catalíticos

La fuerza de infección $(\lambda)$ es una medida propuesta por Hugo Muench (1959), que a diferencia de la tasa de ataque (8), toma en cuenta el cambio de la población entre susceptibles y enfermos. Esta medida es igual a (9)

$$
\lambda=-\log \left[\frac{x(a+1, t+1)}{x(a, t)}\right][1]
$$

donde $x(a, t)$ es la proporción de susceptible a la enfermedad de edad a en el tiempo t. La fuerza de infección tiene una relación inversa con la edad promedio de infección y si el valor de la fuerza de infección es independiente de la edad, la edad promedio de infección es igual a 1/ $\lambda$ (9). Esta medida se relaciona con la seroprevalencia de anticuerpos contra el virus de la enfermedad (10), si esta presenta una baja tasa de mortalidad y el contagio genera inmunidad de por vida. La relación se plantea con base en los siguientes supuestos: La población es estable al igual que su distribución por grupos de edad y la fuerza de infección se puede considerar constante por grupos de edad.

La relación entre la fuerza de infección y la seroprevalencia de anticuerpos contra el VHA se define a partir de los modelos catalíticos $(9,11)$ como se presenta en la fórmula [2]. 


$$
F(a)=1-e^{\left[-\int_{0}^{a} \lambda(\alpha) d \alpha\right]}
$$

Donde F(a) es la seroprevalencia de anticuerpos VHA y $\lambda(a)$ es la fuerza de infección, ambos en función de la edad. Si se asume que el número de infecciones que se presentan en un año tienen una distribución de Poisson con media $\mu$, la fuerza de infección es igual a (12).

$$
\lambda=1-e^{-\mu}[3]
$$

y la fórmula [2] toma la forma de

$$
F\left(A_{i}\right)=1-e^{-\mu A_{i}}
$$

donde Ai es la edad promedio en el grupo i. Si la fuerza de infección es constante por grupos de edad,

$$
\lambda_{i}=1-e^{-\mu_{i}}
$$

para cada grupo de edad.

La estimación de los parámetros del modelo (fórmula [4]) (9) se realiza a través del método de máximo verosimilitud, asumiendo que $\mathrm{F}$ (a) tiene una función de distribución binomial. Para valorar el ajuste del modelos, se utilizó la estadística D (deviance Nelder \& Wedderburn) y la selección del modelo se realizó a partir de la estadística M que es igual a

$$
M=D_{k} / v_{k} \quad[6]
$$

donde vk son los grados de libertad y su valor es igual a n-p, con n el número de grupos de edad y $\mathrm{p}$ el número de parámetros incluidos en el modelo. El modelo con menor valor de la estadística M tendrá un mejor ajuste de los datos. A partir del modelo ajustado, la edad promedio de infección (A) se define como (9)

$$
A \approx \int_{0}^{u}\left[e^{-\mu a}\right] d a \quad[7]
$$

donde $\mathrm{u}$ es la mayor edad de la población bajo estudio y $\mu$ es igual al valor exponencial del parámetro estimado. Si la fuerza de infección sólo 
es constante por grupos de edad, la integral descrita en la fórmula [7] se evalúa por grupo de edad con su correspondiente $\mu_{i}$ estimado.

Para estimar la fuerza de infección de la hepatitis A se realizó el ajuste de los modelos catalíticos (fórmula [4]) a partir de la estimación de la seroprevalencia de anti-VHA para cada ciudad, por los siguientes grupos de edad: De 1 a 4 años, de 5 a 9 años y de 10 a 15 años. Con esta agrupación, se plantearon inicialmente tres modelos para cada ciudad: (Modelo 1) La fuerza de infección es constante en la población de 1 a 15 años, (Modelo 2) la fuerza de infección es constante en el grupo de edad de 1 a 4 años y en el grupo de 5 a 15 años y (Modelo 3) la fuerza de infección es constante en el grupo de 1 a 9 años y en el grupo de 10 a 15 años. Finalmente se seleccionó uno de los tres modelos utilizando la estadística $\mathrm{M}$ y se estimó la fuerza de infección y la edad promedio de infección para cada ciudad. La estimación de los parámetros y sus correspondientes errores de estimación se obtuvieron utilizando la función de optimización (optim u optimize) del programa R 2.8.0 (13).

\section{RESULTADOS}

Estimación de la seroprevalencia de anti-VHA

Se evaluaron 2152 pacientes de 1 a 15 años de edad, en seis instituciones ubicadas en cinco ciudades colombianas, con una edad media de 7,4 años (d.e. $=4,2)$ donde el $51,7 \%$ fueron hombres (Tabla 1). La gran mayoría reside en zona urbana $(99,0 \%$ ) y cuenta con buena cobertura de servicios públicos de acueducto $(99,5 \%)$ y alcantarillado $(99,3 \%)$. El porcentaje de pacientes con anticuerpos positivos fue del $23,9 \%$ en las seis instituciones del país. Se observaron diferencias entre las cinco ciudades con relación a la distribución por grupos de edad. En Barranquilla, el $50 \%$ de su población se encontró en el grupo de 5 a 9 años, mientras que Cali y Bucaramanga presentó mayor porcentaje de su población en el grupo de 10 a 15 años de edad. En Bogotá se observó mayor peso porcentual en el grupo de 1 a 4 años de edad mientras que Medellín presentó una distribución uniforme con porcentajes entre el $32 \%$ y el 34,1 \% en los tres grupos de edad. 


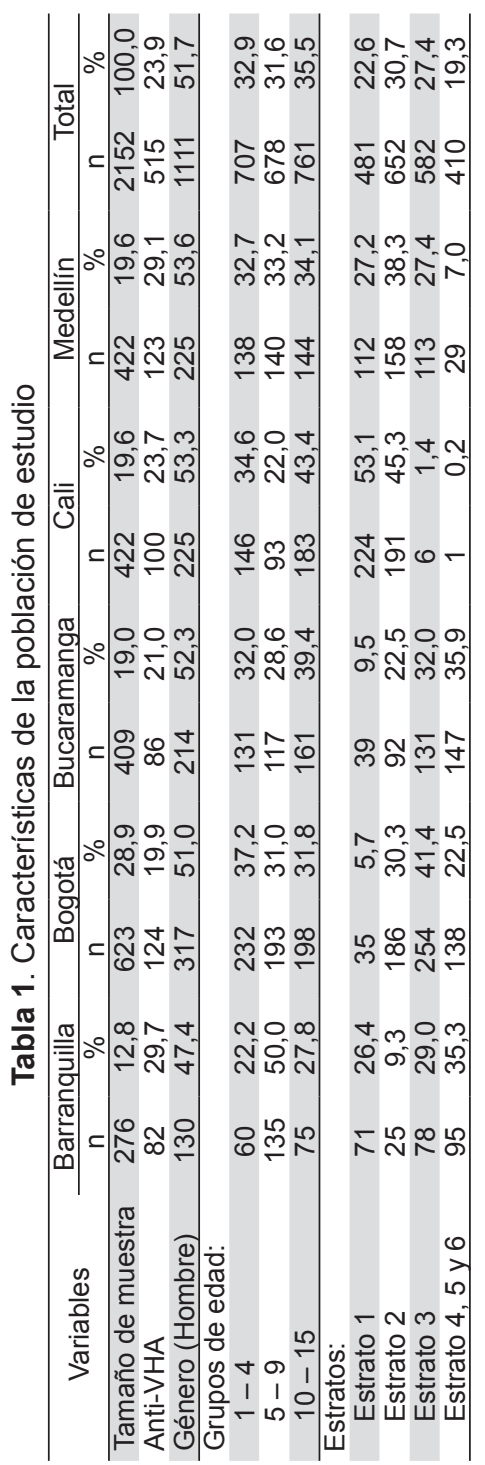




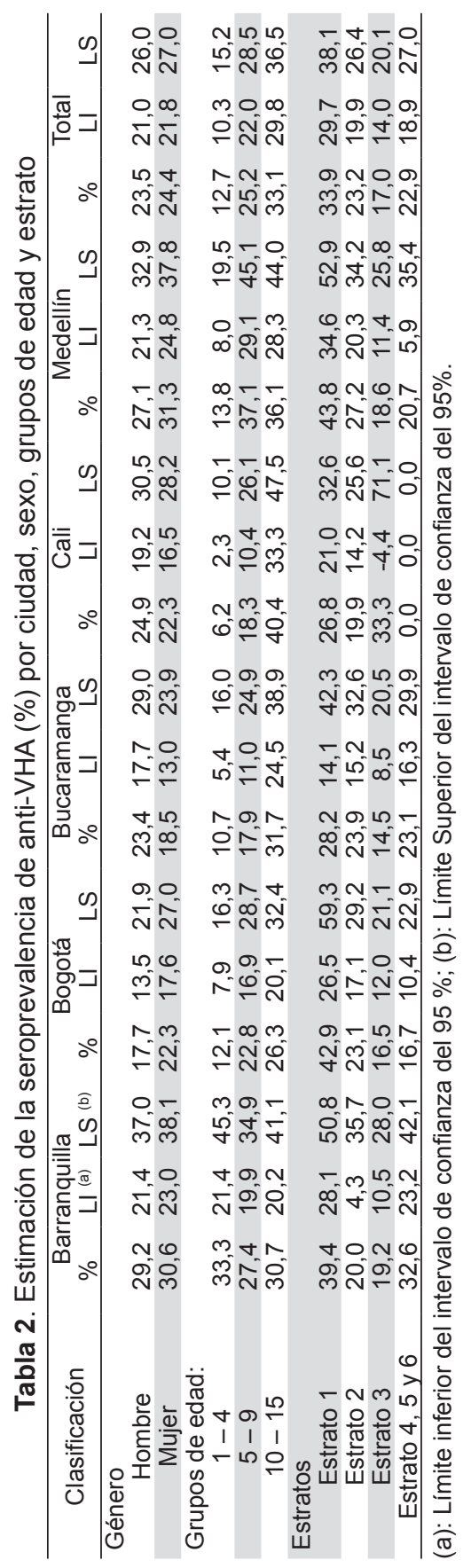


Conrelacióna la distribucióndela población porestratos socioeconómicos por ciudad, en Cali se observó que el 98,3\% de su población se encontró en los estratos 1 y 2, Barranquilla, Bogotá y Bucaramanga presentaron porcentajes entre $64,9 \%$ y $68,0 \%$ en los estratos tres a seis mientras que Medellín presentó el 65,5\% en los estratos 1 y 2 .

Al observar la seroprevalencia de anticuerpos contra el virus de la hepatitis A (Tabla 2) en cada una de las ciudades y comparando sus correspondientes intervalos de confianza, no se observaron diferencias significativas por género. En Barranquilla se observó mayor seroprevalencia en el grupo de 1 a 4 años de edad, mientras que en Bogotá, Bucaramanga y Cali se observó mayor seroprevalencia en los grupos de mayor edad. En Medellín sobresale el alto porcentaje de presencia de anti-VHA en el grupo de 5 a 9 años. En Bogotá, Bucaramanga, Cali y Medellín se presentó mayor seroprevalencia en los estratos más bajos, mientras que en Barranquilla se presentó mayor seroprevalencia en el estrato 1 y en los estratos 4,5 y 6 .

Estimación de la fuerza de infección aplicando modelos catalíticos Para la ciudad de Barranquilla y Bucaramanga, se seleccionó el modelo 2, mientras que en las ciudades de Bogotá, Cali y Medellín el modelo seleccionado fue el número 3. A partir de los modelos seleccionados, se obtuvo para cada ciudad la estimación de: La tasa de infección anual por grupo de edad $\left(\mu_{i}\right)$, la fuerza de infección por grupo de edad $\left(\lambda_{i}\right)$, su error estándar $\left(\operatorname{SE}\left(\lambda_{i}\right)\right)$ y su intervalo con un nivel de confianza del $95 \%$ y la edad promedio de infección (Tabla 3 ).

Tabla 3. Estimación de parámetros, tasa de infección anual, fuerza de infección y edad promedio de infección por ciudad

\begin{tabular}{|c|c|c|c|c|c|c|c|c|c|}
\hline \multirow[b]{2}{*}{ Lugar } & \multirow{2}{*}{$\begin{array}{l}\text { Grupos } \\
\text { de } \\
\text { edad }\end{array}$} & \multirow[b]{2}{*}{$\mathrm{Bi}$} & \multirow[b]{2}{*}{$\mathrm{SE}(\beta \mathrm{i})$} & \multirow[b]{2}{*}{$\mu \mathrm{i}$} & \multirow[b]{2}{*}{$\lambda i$} & \multirow[b]{2}{*}{$\operatorname{SE}(\lambda i)$} & \multicolumn{2}{|c|}{ I.C. 95\% para $\lambda \mathrm{i}$} & \multirow{2}{*}{$\begin{array}{c}\text { Edad } \\
\text { promedio } \\
\text { infección }\end{array}$} \\
\hline & & & & & & & $\begin{array}{l}\text { Límite } \\
\text { Inferior }\end{array}$ & $\begin{array}{l}\text { Límite } \\
\text { Superior }\end{array}$ & \\
\hline \multirow{2}{*}{ Barranquilla } & $1-4$ & $-1,82$ & 0,23 & 0,16 & 0,15 & 0,031 & 0,089 & 0,210 & \multirow{2}{*}{ NA } \\
\hline & $5-15$ & $-3,31$ & 0,13 & 0,04 & 0,04 & 0,005 & 0,027 & 0,045 & \\
\hline \multirow{2}{*}{ Bogotá } & $1-9$ & $-3,23$ & 0,12 & 0,04 & 0,04 & 0,004 & 0,030 & 0,048 & \multirow{2}{*}{11,9} \\
\hline & $10-15$ & $-3,71$ & 0,14 & 0,02 & 0,02 & 0,003 & 0,018 & 0,031 & \\
\hline \multirow{2}{*}{ Bucaramanga } & $1-4$ & $-3,10$ & 0,27 & 0,05 & 0,04 & 0,012 & 0,022 & 0,067 & \multirow{2}{*}{12,0} \\
\hline & $5-15$ & $-3,54$ & 0,12 & 0,03 & 0,03 & 0,003 & 0,022 & 0,035 & \\
\hline & $1-9$ & $-3,6$ & 0,20 & 0,03 & 0,03 & $0,0 c$ & 0,016 & 0,036 & \multirow{2}{*}{$11, \varepsilon$} \\
\hline & $10-15$ & $-3,18$ & 0,12 & 0,04 & 0,04 & 0,005 & 0,031 & 0,050 & \\
\hline \multirow{2}{*}{ Medellín } & $1-9$ & $-2,79$ & 0,12 & 0,06 & 0,06 & 0,007 & 0,046 & 0,073 & \multirow[b]{2}{*}{10,7} \\
\hline & $10-15$ & $-3,33$ & 0,14 & 0,04 & 0,04 & 0,005 & 0,026 & 0,045 & \\
\hline
\end{tabular}


En Barranquilla se observó una gran diferencia en la estimación de la fuerza de infección para los dos grupos de edad seleccionados, debido a que en el grupo de 1 a 4 años, el 91,7\% corresponde a los estratos 1 y 2 , mientras que en el grupo de 5 a 15 años el mayor peso se encontró en los estratos de 3, 4, 5 y 6 con el 81,0\%. Por lo anterior, se decidió no realizar la estimación de la edad promedio de infección para Barranquilla, considerando que el resultado no representaría a ninguna de las poblaciones de los dos grupos de edad.

En la Figura 1 se observa la seroprevalencia de anti-VHA estimada por el modelo seleccionado (línea) y la seroprevalencia observada en el punto medio de intervalo de edad (barra) para cada ciudad.

Únicamente en Cali hubo un comportamiento con crecimiento constante en la estimación de la seroprevalencia de anti-VHA, lo cual es consistente con el supuesto de una población estable. En el resto de ciudades, se observaron disminuciones de un año a otro en un punto que se encuentra relacionado con el modelo seleccionado para cada ciudad.

Figura 1. Seroprevalencia de anti-VHA estimada vs.

Seroprevalencia observada en cinco ciudades colombianas

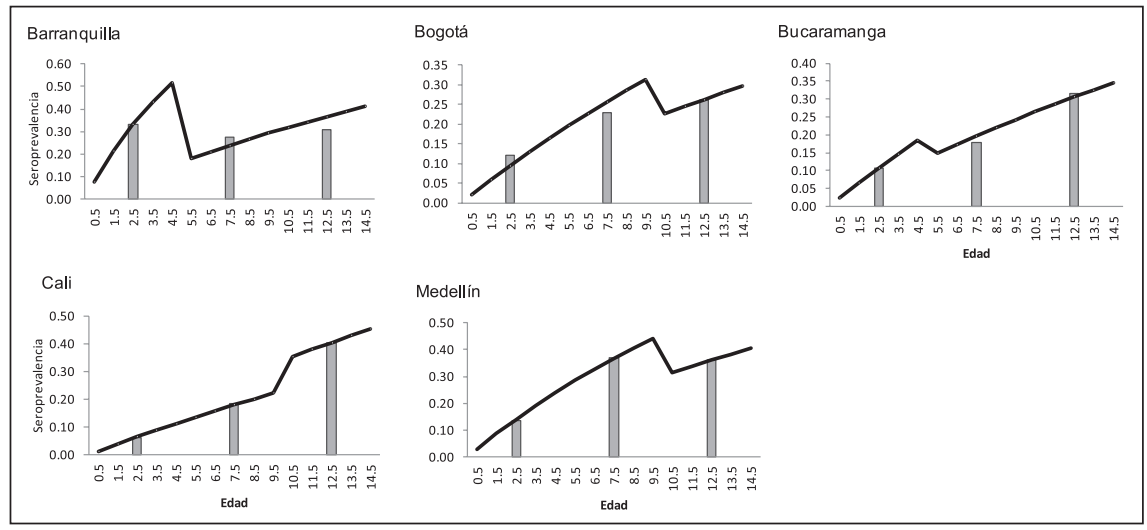

\section{DISCUSIÓN}

La fuerza de infección estimada para Barranquilla, Bogotá, Bucaramanga y Medellín, fueron significativamente mayores a lo reportado por una ciudad desarrollada como Madrid (España) (12), donde la fuerza de 
infección para el grupo de niños entre los 2 y los 11 años de edad fue de 0,017 [I.C. $95 \%$ de 0,013-0,02].

Utilizando los resultados de seroprevalencia publicados de las ciudades de Santiago (14), Lima (7), Montevideo (3), Caracas (5) y Buenos Aires y Córdoba (Argentina) (5), se ajustaron los modelos catalíticos para estimar la fuerza de infección y la edad promedio de infección para estos lugares. Se tomaron los grupos de edad de 1 a 15 años y se ajustaron para cada ciudad, los tres modelos propuestos inicialmente para nuestra población. La selección del modelo para cada lugar se realizó a partir de la estadística D y M. Los tres modelos planteados para el reporte de Argentina no pasaron la prueba de bondad de ajuste $(p<0,05)$, por este motivo no se incluyó en la comparación. En la Tabla 4 se presenta la fuerza de infección y la edad promedio de infección obtenida para Santiago de Chile, Lima, Montevideo y Caracas.

La fuerza de infección estimada en la ciudad de Barranquilla en el grupo de edad de 1 a 4 años fue igual 0,15 , valor cercano al obtenido en Lima para este grupo de edad. Los valores de la fuerza de infección estimados para Bogotá, Bucaramanga, Cali y Medellín son similares a los obtenidos en Santiago y Montevideo, al igual que su edad promedio de infección, sugiriendo un nivel de endemicidad intermedia según la clasificación propuesta por la OMS. Con relación a Lima y Caracas, las cuatro ciudades colombianas presentaron una fuerza de infección menor y una edad promedio de infección mayor.

Tabla 4. Fuerza de infección y edad promedio de infección para cuatro ciudades de Latinoamérica

\begin{tabular}{cccc}
\hline Lugar & Grupo de edad & $\lambda$ & $\begin{array}{c}\text { Edad promedio } \\
\text { de infección }\end{array}$ \\
\hline Caracas - Venezuela & $1-10$ & 0,09 & 8,5 \\
Lima - Perú & $11-15$ & 0,07 & 8,0 \\
Montevideo - Uruguay & $1-4$ & 0,13 & 10,7 \\
Santiago - Chile & $5-14$ & 0,07 & 10,3 \\
\hline
\end{tabular}

En Bucaramanga y Cali aunque los modelos seleccionados consideraron dos parámetros, tanto la prueba de bondad de ajuste como la comparación de los intervalos de confianza, no reflejaron diferencias significativas en la 
fuerza de infección estimada entre el intervalo de 1 a 15 años de edad, esta misma situación se presentó en Santiago de Chile y en Montevideo, donde se observaron fuerzas de infección entre 0,04 y 0,05 y la prueba de bondad de ajuste no rechazó el modelo 1.

La seroprevalencia estimada para Barranquilla, Bogotá, Bucaramanga y Medellín presentaron una disminución entre el rango de 1 y 15 años de edad, lo anterior se puede presentar por: 1. Cambios en la fuerza de infección al interior de los grupos de edad que no pudieron ser detectados, 2. Presencia de variables diferentes a la edad y el estrato socioeconómico que tienen un impacto en la estimación de la seroprevalencia de anti-VHA o 3. La población bajo estudio no es estable y su movilidad afecta la estimación de seroprevalencia en los grupos de edad considerados en este trabajo. Con relación a la estimación de la edad promedio de infección para cada región, se debe considerar que ésta se ve afectada por la falta de información de la seroprevalencia de anti-VHA de personas mayores de 15 años, que al no ser incluidos en la muestra, la estimación tiende a tomar un menor valor.

Aunque la selección de la muestra no se realizó a partir de un muestreo probabilístico, en ella se incluyen niños de los seis estratos socioeconómicos y de los tres grupos de edad, lo que permite acercarnos a la seroprevalencia de las zonas urbanas de las ciudades de Bogotá, Bucaramanga, Cali y Medellín. Finalmente, se resalta la importancia de seguir trabajando en la caracterización de la seroprevalencia de anti-VHA para otras ciudades del país, buscando apoyar la implementación de esquemas de vacunación apropiados para cada región

\section{REFERENCIAS}

1. Rodríguez-Acosta C. Actualización sobre hepatitis viral: Etiología, patogenia, diagnóstico microbiológico y prevención. Rev. Cubana Med Gen Integr. 2000; 16(6):574-85.

2. Vargas V, González A. Hepatitis A. En: Guardia J, Esteban R. Monografías Clínicas. Enfermedades Infecciosas; 2. Hepatitis Vírica. Barcelona: Doyma; 1988. pp. 5-16.

3. Montano A, Barañano R, Lageard B, Moratorio G, Dibarboure H, García A, Gonzalez M, Pírez MC, Russi JC, Chiparelli H, Ferrari AM, Zballos E, Dibarboure M, Suarez N, Giambruno G, Fazzio S. Prevalencia de hepatitis A en niños de 2 a 14 años y en población laboral de 18 a 49 años en Montevideo, Uruguay. Rev. Med. Uruguay. 2001; 17:84-98.

4. Wolfgang J, Deinhardt F, Hilleman M. Hepatitis A Vaccine. Vaccine. 1994; 20:583-95.

5. Tapia-Conyer R, Santos JI, Cavalcanti AM, Urdaneta E, Rivera L, Manterola A, et al. Hepatitis A in Latin America: a changing epidemiologic pattern. Am J Trop Med Hyg. 1999; 61:825-829. 
6. Armstrong GL, Bell BP. Hepatitis A virus infections in the United States: model-based estimates and implications for childhood immunization. Pediatrics. 2002;109:839-845.

7. Vildósola $H$, Colichón A, Rubio MP, Weil J. Prevalencia de anticuerpos contra hepatitis A (anti-HVA IgG) en una población de 01 a 39 años en Lima. Revista de Gastroenterología del Perú. 2000; 20 (2): 141-145.

8. Kleinbaum D, Kupper L, Morgenstern H. Epidemiology Research. Principles and quantitative methods. Capítulos 6. New York: Van Nostrand Reinhold; 1982.

9. Grenfell BT, Anderson RM. The estimation of age-related rates of infection from case notifications and serological data. The Journal of Hygiene; 1985; 95 (2): 419-436.

10. Muench H. Catalytics Models in Epidemiology. Harvard: Harvard University Press; 1959.

11. Griffiths DA. A Catalytic Model of Infection for Measles. Applied Statistics. 1974; 23 (3): 330-339.

12. Amela C, Pachón I, Bueno R, De Miguel C, Martinez-Navarro F. Trends in hepatitis A virus infection with reference to the process of urbanization in the greater Madrid area (Spain). European Journal of Epidemiology. 1995; 11 (5): 569-573.

13. Marco RS [Internet]. Maximum Likelihood Programming in R. Enero 2006. Disponible en: http://www.unc.edu/ monogan/computing/r/MLE_in_R.pdf Consultado 10 de abril de 2009.

14. Alan DF, Oriana SM, Gallicchio L, Pablo AV, Lagos R. Age-specific prevalence of antibodies to hepatitis a in Santiago, Chile: Risk factors and shift in age of infection among children and young adults. Am. J. Trop. Med. Hyg. 2002; 66 (5): 628-632. 